\title{
Effects of oxygen-ozone therapy in the Roth syndrome: a case report
}

\author{
Alfredo Romeo, ${ }^{1,2}$ Carolina Baiano, ${ }^{3}$ Marianna Chierchia, ${ }^{4}$ Francesco Scandone ${ }^{5}$ \\ ${ }^{1}$ Department of Physical and Rehabilitation Medicine, "Umberto I" Hospital, Siracusa; ${ }^{2}$ Italian Society for Oxygen-Ozone \\ Therapy (SIOOT), Gorle (BG); ${ }^{3}$ Physical and Rehabilitation Medicine Health District, Provincial Health Authority, \\ Siracusa; "Luigi Vanvitelli" University of Campania; "A. Landolfi" Pain Control Center, Solofra (AV), Italy
}

\begin{abstract}
Meralgia paresthetica (MP) is a disesthetic and/or anesthetic syndrome in the distribution of the lateral femoral cutaneous nerve. It is a compressive or traumatic mononeuropathy, characterized by burning pain and/or discomfort in the anterolateral thigh, without motor or muscle strength changes, with preserved reflexes. The authors report the case of a 52-year-old male with a history of low back pain presented from two-year sensory dysesthesias and paresthesias in the right anterolateral thigh, consistent with meralgia paresthetica; they also describe the first reported use of $\mathrm{O}_{2}-\mathrm{O}_{3}$ therapy to relieve the intractable pain associated with MP.
\end{abstract}

\section{Case Report}

A 52-year-old male with a history of low back pain presented from two-year sensory dysesthesias and paresthesias in the right anterolateral thigh, consistent with meralgia paresthetica (MP). Temporary relief occurred with multiple lateral femoral cutaneous nerve (LFCN) and fascia lata blocks at 2 different institutions. The patient expressed dissatisfaction with his previous treatments and requested any therapeutic intervention that might lead to long-lasting pain relief. At this time, $\mathrm{O}_{2}-\mathrm{O}_{3}$ therapy was

Correspondence: Alfredo Romeo, Italian Society for Oxygen-Ozone Therapy (SIOOT), Via Don Luigi Sturzo 2, 24020 Gorle (BG), Italy. E-mail: alfredo.romeo@tin.it

Key words: Roth syndrome; Meralgia paresthetica; Lateral femoral cutaneous nerve; Oxygen-ozone therapy.

Conflict of interest: the authors declare no potential conflict of interest.

Received for publication: 3 December 2018.

Accepted for publication: 5 December 2018.

C) Copyright A. Romeo et al., 2018

Licensee PAGEPress, Italy

Ozone Therapy 2018; 3:7970

doi:10.4081/ozone.2018.7970

This article is distributed under the terms of the Creative Commons Attribution Noncommercial License (by-nc 4.0) which permits any noncommercial use, distribution, and reproduction in any medium, provided the original author(s) and source are credited. then undertaken with infiltration in the paravertebral region L2L3 and L3-L4 at the concentration of $20 \mathrm{ng} / \mathrm{mL}$ with $23 \mathrm{G}$ needle for 8 sessions, at the same time subcutaneous microinjections in anterior upper anterior region of the right thigh at a concentration of $10 \mathrm{ng} / \mathrm{mL}$ with $30 \mathrm{G}$ needle for 8 sessions were administered (the first 4 twice a week, the subsequent weekly and then fortnightly). The patient reported exceptional pain relief yet from the second infiltration and prolonged at 12-month followup. The patient had experienced long-standing pain that was recalcitrant to conservative/pharmacologic therapy and multiple nerve blocks with local steroid instillations. A single cycle with $\mathrm{O}_{2}-\mathrm{O}_{3}$ therapy resulted in complete and sustained cessation of pain. No side effects were evident. $\mathrm{O}_{2}-\mathrm{O}_{3}$ therapy of the LFCN may offer an effective, low risk treatment in patients with MP who are refractory to conservative medical management or are unwilling or unfit to undergo surgery.

\section{Discussion and Conclusions}

MP, mononeuropaty of the LFCN, is characterized by symptoms of pain, numbness, itching and parasthesia at the antero-lateral thigh. Hager et al. first reported its symptoms in 1885 and eventually named by Roth in $1895 .{ }^{1}$ Bernhardt et al. presumed that the symptoms were caused by compression of the LFCN. ${ }^{1}$

The LFCN is part of the lumbar plexus. It functions primarily as a sensory nerve and its composition varies among individuals with several different combinations of lumbar nerves that originate from L1 to L3. The LFNC then emerges at the lateral border of the psoas major, crosses the iliacus, to the anterior superior iliac spine. The nerve then passes under the inguinal ligament and over the sartorius muscle and enters the thigh as it divides into an anterior and posterior branch.

This anatomical course has traditionally been accepted by researchers and clinicians. However, researchers have demonstrated variability in the nerve's course as it exits the pelvis. Azmann et al. have classified 5 different variations in the LFNC discovered through their cadaveric investigation. ${ }^{1}$ These variations have also been documented by other investigators. Compression of the LFCN most commonly occurs as it exits the pelvis. The anatomical variations may clinically present with different signs and symptoms due to the unique course the nerve may take as it provides sensory innervation to the anterolateral thigh. A working knowledge of these potential variations can be useful for the clinician during their examination as well as when establishing and or making adjustments to a plan of care. ${ }^{1}$

MP has an incidence of 4-10/10,000 people and usually occurs in those aged 30-40 years. It is commonly caused by mechanical pressure exerted on the LFCN, such as a pressure 
from wearing thigh underwear or a lumbar brace and from prone positioning.

During its course, but mostly close to the ASIS, the nerve can be injured or compressed, provoking the typical symptoms of MP: anesthesia or ipoesthesia of the lateral thigh and gluteal region, pain or paresthesia.

Grossman et al. classified MP as being idiopathic or iatrogenic with a sub classification of idiopathic as mechanical or metabolic. ${ }^{2}$

\section{Idiopathic meralgia paresthetica}

Mechanical factors can result in compression of the LFCN along its anatomical course. MP has been related to the following factors: obesity $(\mathrm{BMI} \geq 30)$, pregnancy, tight garments such as jeans, military armor and police uniforms, seat belts, direct trauma, muscle spasm, scoliosis, iliacus hemotoma, and leg length changes. Metabolic factors reported include diabetes mellitus, alcoholism, and lead poisoning.

\section{Iatrogenic meralgia paresthetica}

MP has also been reported as a post-surgical complication after hip joint replacement and spine surgery. MP has also been reported, to a lesser extent, as a post-surgical complication in iliac bone harvesting, open and laparoscopic appendectomy, caesarean with epidural analgesics, and obstetric and gynaecological surgery.

The etiopathogenetic hypothesis that we are advancing is that in reality the origin of the symptomatology is a canalicular origin and a vertebral origin, one speaks therefore of mixed origin. It is called canalicular the suffering of a compression nerve in its passage in an anatomical structure with a tunnel that is obliged, restricted and extensible. The nerves in their course, often have to overcome the bottleneck, through canalicular formations or hosts that generally are constituted by a fibrous tissue that originates from the aponeurosis. In these strains the nerve first begins to suffer with an irritation of the perineurium, then with a suffering of the nerve fibers in which the motor and/or sensory deficit occurs spontaneously without necessarily a traumatic mechanism.

The vertebral origin would be due to a degenerative disc and/or arthrosic pattern of the L2-L3 segment; the patient sometimes complains of low back pain on the same side of meralgia, but not necessarily low back pain is concomitant and usually passes into the background.

The mixed origin, therefore, is when both a canalicular and vertebral suffering occur simultaneously. The success of treatment with $\mathrm{O}_{2}-\mathrm{O}_{3}$ infiltration, both in the paravertebral region L2-L3 and L3-L4 and in anterior upper anterior region of the right thigh, confirms our hypothesis. We also believe that paresthesia meralgia can be counted among the Double crush syndrome.

Double crush syndrome is a distinct compression at two or more locations along the course of a peripheral nerve that can coexist and synergistically increase symptom intensity. In addition, dissatisfaction after treatment at one site may be the result of persistent pathology at another site along a peripheral nerve. Double crush syndrome is a controversial diagnosis; some scientists and surgeons believe it is an illness construction that may do more harm than good because it emphasizes an objective pathophysiologic explanation for unexplained symptoms, disability, and dissatisfaction that may be more psychosocially mediated. However, peripheral neuropathy may coexist with compressive neuropathy and contribute to suboptimal outcomes following nerve decompression. ${ }^{3}$

MP usually improves by conservative treatment, however surgical treatment such as neurolysis can be useful in some cases of intractable pain; we describe the first reported use of $\mathrm{O}_{2}-\mathrm{O}_{3}$ therapy to relieve the intractable pain associated with MP.

\section{References}

1. Cheatham SW, Kolber MJ, Salamh PA. Meralgia paresthetica: a review of the literature. Int J Sports Phys Ther 2013;8:883-93.

2. Grossman MG, Ducey SA, Nadler SS, et al. Meralgiaparesthetica: diagnosis and treatment. J Am Acad Orthop Surg 2001;9:336-44.

3. Kane PM, Daniels AH, Akelman E. Double crush syndrome. J Am Acad Orthop Surg 2015;23:558-62. 